\title{
Determination of the properties of vector mesons in external magnetic field by quenched $\mathrm{SU}(3)$ lattice QCD
}

\author{
E.V. Luschevskaya, ${ }^{a, b}$ O.E. Solovjeva ${ }^{a}$ and O.V. Teryaev ${ }^{c, d}$ \\ ${ }^{a}$ Institute for Theoretical and Experimental Physics \\ named by A.I. Alikhanov of NRC "Kurchatov Institute", \\ 117218, Bolshaya Cheremushkinskaya 25, Moscow, Russia \\ ${ }^{b}$ Moscow Institute of Physics and Technology, \\ Dolgoprudnyj, Institutskij lane 9, Moscow Region 141700, Russia \\ ${ }^{c}$ Joint Institute for Nuclear Research, \\ Dubna, 141980, Russia \\ ${ }^{d}$ Lomonosov Moscow State University, \\ GSP-1, Leninskie Gory, 119991 Moscow, Russia \\ E-mail: luschevskaya@itep.ru, olga.solovjeva@itep.ru, \\ teryaev@theor.jinr.ru
}

ABSTRACT: We investigate the ground state energies of vector $\rho^{ \pm}$and $K^{ \pm *}$ mesons depending on the magnetic field value in the $\mathrm{SU}(3)$ lattice gauge theory. It has been shown that the energy of a vector particle depends on its spin projection on the field axis. The magnetic dipole polarizability and hyperpolarizabilities give significant contributions to the energy value which prevents the formation of the charged vector meson condensate at high magnetic fields. We calculate the g-factor of $\rho^{ \pm}$and $K^{ \pm *}$ mesons and the dipole magnetic polarizability of $\rho^{ \pm}$mesons.

KEYwords: Lattice QCD, Lattice Quantum Field Theory, Nonperturbative Effects

ARXIV EPRINT: 1608.03472 


\section{Contents}

1 Introduction 1

2 Details of calculations 2

2.1 Fermionic spectrum 2

2.2 Gauge field action 3

2.3 Abelian magnetic field on the lattice 3

2.4 Calculation of correlation functions 4

3 Magnetic moments of $\rho^{ \pm}$and $K^{* \pm}$ mesons $\quad 7$

4 Energy of $\rho^{ \pm}$meson 11

$5 \quad$ Magnetic polarizabilities of $\rho$ meson $\quad 12$

$\begin{array}{llr}6 & \text { Conclusion } & 18\end{array}$

$\begin{array}{ll}\text { A Lattice parameters and values of the magnetic field } & 19\end{array}$

\section{Introduction}

Magnetic fields of hadronic scale could exist in cosmic objects and Early Universe. The noncentral heavy ion collisions may create such fields in terrestrial laboratories like LHC (ALICE), RHIC, NICA and FAIR (CBM) [1]. The sufficiently strong magnetic field affects the internal structure of hadrons, in particular shifts their energy levels.

Since a meson is a composite particle, the external electromagnetic field will cause the deformation of the meson wave function. The way the meson is deformed under the action of the external field is determined by the properties of the strong interaction between the quark and the antiquark.

In this work we consider the constant external magnetic field. The magnetic moments, the dipole magnetic polarizability and the hyperpolarizabilities characterize the response of a particle to this external influence. The magnetic moment is the most important quantity describing the magnetic properties of a meson or baryon due to the presence of the spin. The magnetic polarizabilities and hyperpolarizabilities show the distribution of the quark currents inside an hadron and describe its internal structure in the external magnetic field.

The effects of a magnetic field on an hadron mass were firstly studied in the pioneering paper [2]. Then the magnetic field was introduced in two flavor lattice QCD simulation [3] and used as the probe of QCD properties [4]. The energies of hadrons in magnetic fields have been calculated on the lattice [5-8], in theoretical models [9-13] and in the framework of the QCD sum rules $[14,15]$. The notion of the hadron polarizability was initially 
discussed in $[16,17]$. The magnetic polarizabilities of pions have been measured in the experiments [18-20], have been calculated in the chiral perturbation theory [21, 22] and in the lattice gauge theory [23]. The magnetic polarizabilities of baryons have been obtained in full lattice QCD [8]. The magnetic moment of the $\rho$ meson has been explored in [24-30].

This work is devoted to exploration of the energy levels of $\rho$ and $K^{*}$ mesons in the magnetic field, their magnetic polarizabilities and magnetic moments. Our results were obtained in pure $\mathrm{SU}(3)$ lattice gauge theory. The inclusion of dynamical quarks into a consideration will most likely not lead to a radical change in the magnetic properties of $\rho$ meson, although it becomes an unstable particle in full QCD. For example, in $2+1$ full QCD the g-factor of the $\rho^{ \pm}$meson is equal to 2.21(8) at the physical point [30]. Quenched lattice theory predicts the value $g=2.25(0.34)$ in the chiral limit [24] and $g=2.20(0.15)$ at the lowest pion mass [27].

In section 2 we describe the technical details of our calculations, the gauge field action, the fermionic spectrum and correlation functions. In section 3 the energy of the charged vector $\rho$ meson has been studied for various spin projections versus the magnetic field value. Section 4 is devoted to the discussion of the magnetic moments of the $\rho^{ \pm}$and $K^{* \pm}$ mesons. The magnetic dipole polarizability and hyperpolarizability of the first order of the $\rho^{ \pm}$mesons have been calculated in section 5 .

\section{Details of calculations}

\subsection{Fermionic spectrum}

To calculate the eigenvalues and the eigenvectors of the Dirac operator we use the Neuberger overlap operator [31]. It has the following form

$$
D_{\mathrm{OV}}=\frac{\rho}{a}\left(1+\frac{D_{W}}{\sqrt{D_{W}^{\dagger} D_{W}}}\right)=\frac{\rho}{a}\left(1+\gamma_{5} \operatorname{sign}(H)\right),
$$

where $D_{W}=M-\rho / a$ is the Wilson-Dirac operator with the negative mass term $\rho / a$, $\rho=1.4$ is the parameter, $H=\gamma_{5} D_{W}$ is the hermitian Wilson-Dirac operator, $a$ is the lattice spacing in physical units, $M$ is the Wilson hopping term with $r=1$. The key ingredient of the overlap operator is the $\operatorname{sign}(H)$ function

$$
\operatorname{sign}(H)=\frac{H}{\sqrt{H^{\dagger} H}}
$$

Let's construct the massive overlap operator

$$
M_{\mathrm{OV}}=\left(1-\frac{a m_{q}}{2 \rho}\right) D_{\mathrm{OV}}+m_{q}
$$

for the quark mass $m_{q}$.

For the numerical implementation of this operator the MinMax polynomial approximation of the Sign function is used [32]. The spectrum of the Dirac operator $\operatorname{spec}(H) \in$ 
$\left[\lambda_{\min }, \lambda_{\max }\right] \in \mathcal{R}$. The Sign function also can be expressed through the norm $\|H\|$

$$
\operatorname{sign}(H)=\operatorname{sign}\left(\frac{H}{\|H\|}\right)=\operatorname{sign}(W) .
$$

Since $\|H\|=\lambda_{\max }, \operatorname{spec}(W) \in\left[\lambda_{\min } / \lambda_{\max } ; 1\right]$. The function $1 /\|H\|$ is approximated by the Chebyshev polynomials $T_{k}(z), k=0, \ldots, n$ on the interval $\sqrt{\epsilon} \leqslant \operatorname{spec}(H) \leqslant 1$, where $\epsilon=\lambda_{\min }^{2} / \lambda_{\max }^{2}$. The original matrix $H$ and the polynomial

$$
P_{n}\left(H^{2}\right)=\sum_{k=0}^{n} c_{k} T_{k}(z), \quad z=\frac{2 H^{2}-1-\epsilon}{1-\epsilon},
$$

has the same set of eigenfunctions $\psi_{k}$ [33].

The maximal relative error is

$$
\delta=\max |h(y)|
$$

where

$$
h(y)=\frac{1 /\|H\|-P_{n}\left(H^{2}\right)}{1 /\|H\|},
$$

in our calculations $\delta \sim 10^{-8}$. The fermionic propagators are calculated using the eigenfunctions and the eigenvalues of the overlap Dirac operator. This method controls the computational errors with a high efficiency and preserves the chiral invariance at a zero quark mass on the lattice [32].

\subsection{Gauge field action}

We generate ensembles of 200-350 statistically independent quenched SU(3) configurations of the gauge field using the tadpole improved Lüscher-Weisz action [34].

$$
S=\beta_{\mathrm{imp}} \sum_{\mathrm{pl}} S_{\mathrm{pl}}-\frac{\beta_{\mathrm{imp}}}{20 u_{0}^{2}} \sum_{\mathrm{rt}} S_{\mathrm{rt}},
$$

where $S_{\mathrm{pl}, \mathrm{rt}}=(1 / 2) \operatorname{Tr}\left(1-U_{\mathrm{pl}, \mathrm{rt}}\right)$ is the plaquette (denoted by $\mathrm{pl}$ ) or $1 \times 2$ rectangular loop term $(\mathrm{rt}), u_{0}=\left(W_{1 \times 1}\right)^{1 / 4}=\left\langle(1 / 2) \operatorname{Tr} U_{\mathrm{pl}}\right)^{1 / 4}$ is the input tadpole factor computed at zero temperature [35]. This action suppresses ultraviolet dislocations which leads to non-physical near-zero modes of the Wilson-Dirac operator and difficulties in choice of the $\rho$ parameter (see subsection 2.1).

\subsection{Abelian magnetic field on the lattice}

We consider charged particles in a constant external magnetic field. The Abelian magnetic vector potential $A_{\mu}$ is chosen in the symmetric gauge

$$
A_{\mu}^{B}(x)=\frac{B}{2}\left(x_{1} \delta_{\mu, 2}-x_{2} \delta_{\mu, 1}\right) .
$$

This insertion does not affect the dynamics of the gluon as we consider the quenched approximation. The resulting gauge field is presented as the sum

$$
A_{\mu i j}=A_{\mu i j}^{g l}+A_{\mu}^{B} \delta_{i j}
$$


of the nonabelian SU(3) gauge field of gluons $A_{\mu i j}^{g l}$ and the U(1) field of the magnetic photons $A_{\mu}^{B} ; i, j=1, \ldots, N_{c}^{2}-1, \mu=1,2,3,4$ are the color and the Lorentz indices respectively.

In order to respect the gauge invariance of the theory on the lattice and satisfy the periodic boundary conditions in space one has to impose the twisted boundary conditions on fermionic fields [36]. In our case they have the following form

$$
\begin{aligned}
& \psi\left(x_{1}+a N_{s}, x_{2}, x_{3}\right)=\exp \left(-i \frac{q}{2} B a N_{s} x_{2}\right) \psi\left(x_{1}, x_{2}, x_{3}\right), \\
& \psi\left(x_{1}, x_{2}+a N_{s}, x_{3}\right)=\exp \left(i \frac{q}{2} B a N_{s} x_{1}\right) \psi\left(x_{1}, x_{2}, x_{3}\right),
\end{aligned}
$$

where $N_{s}$ is the number of lattice sites in spatial directions, $q$ is the quark charge. There is also an additional gauge dependence of the electromagnetic gauge potential and fermion field, which is analogous to the $\theta$-vacuum in QCD [36].

The non-Abelian gauge $\mathrm{SU}(N)$ theories in a box with an external electromagnetic field and periodic boundary conditions were originally investigated in [37]. The Landau level problem has been studied on a torus in papers [38, 39]. The condition of quantizing for the magnetic flux can be also derived from consistency of the boundary conditions (2.11) and (2.12), see [36]. Therefore the magnetic field value is determined by the relation

$$
q B=\frac{2 \pi n_{B}}{\left(a N_{s}\right)^{2}}, \quad n_{B} \in \mathbb{Z},
$$

where $q=-1 / 3 e$ is the charge of the $d$-quark, $N_{s}$ is the size of the lattice in the spatial direction. We explore the sufficiently large magnetic fields corresponding to $n_{B} \sim 0 \div 28$, where the saturation regime $\left(n_{B} /\left(L^{2}\right) \gtrsim 0.5\right)$ is not yet achieved. The radius of the first Landau level $l_{H}=1 / \sqrt{e B}$ is also larger for the range of fields and lattice spacings presented here. The lattice parameters and the values of the magnetic field used for the calculations are presented in appendix.

\subsection{Calculation of correlation functions}

The correlation functions of the vector fermionic currents for the charged mesons in the coordinate space

$$
\left\langle O_{\rho^{+}}(x) \bar{O}_{\rho^{+}}(y)\right\rangle=-\operatorname{Tr}\left[\Gamma_{1} D_{u}^{-1}(x, y) \Gamma_{2} D_{d}^{-1}(y, x)\right],
$$

where $O_{\rho^{+}}=\psi^{\dagger}(x)_{d} \Gamma_{1,2} \psi(x)_{u}$ is the $\rho^{+}$meson interpolator, $\Gamma_{1}, \Gamma_{2}=\gamma_{\mu}$ are the gamma matrices, $D_{u, d}^{-1}$ are the propagators of $u$ and $d$ quarks. The interpolation operator for the $\rho^{-}$ meson is defined similarly, $O_{\rho^{-}}=\psi^{\dagger}(x)_{u} \Gamma_{1,2} \psi(x)_{d}$. The lattice interpolation operator for the $\pi^{0}$ meson has the form $O_{\pi^{0}}=\left(\psi^{\dagger}(x)_{u} \gamma_{5} \psi(x)_{u}-\psi^{\dagger}(x)_{d} \gamma_{5} \psi(x)_{d}\right) / \sqrt{2}$. The corresponding correlation function reads

$$
\left\langle O_{\pi^{0}}(x) \bar{O}_{\pi^{0}}(y)\right\rangle=-\frac{1}{2} \operatorname{Tr}\left[\gamma_{5} D_{u}^{-1}(x, y) \gamma_{5} D_{u}^{-1}(y, x)\right]-\frac{1}{2} \operatorname{Tr}\left[\gamma_{5} D_{d}^{-1}(x, y) \gamma_{5} D_{d}^{-1}(y, x)\right],
$$

where only the connected part is included. The disconnected part is zero because the $u$ and $d$ quarks contributions cancel each other. 
The massive Dirac propagator in an external magnetic field is expressed as the sum over the lowest $M$ Wilson-Dirac eigenmodes

$$
D^{-1}(x, y)=\sum_{k<M} \frac{\psi_{k}(x) \psi_{k}^{\dagger}(y)}{i \lambda_{k}+m},
$$

where $x=\left(\mathbf{n} a, n_{t} a\right)$ and $y=\left(\mathbf{n}^{\prime} a, n_{t}^{\prime} a\right)$ are the coordinates on the lattice. $\mathbf{n}, \mathbf{n}^{\prime} \in \Lambda_{3}=$ $\left\{\left(n_{1}, n_{2}, n_{3}\right) \mid n_{i}=0,1, \ldots, N_{s}-1\right\}$ denote the numbers of lattice sites in the spatial directions, $n_{t}, n_{t}^{\prime}$ are the numbers of lattice sites in the time direction. We use $M=50$ to provide the high level of convergence.

We carry out the Fourier transformation of our meson interpolators to the momentum space over the spatial components $\mathbf{n}$ in the spatial lattice $\Lambda_{3}$. Then the interpolators are projected to zero spatial momentum $\mathbf{p}=0$ which corresponds to the meson at rest. At zero magnetic field the meson energy equals to its mass $E_{0}=m_{0}$.

The correlation function in the Euclidean space can be represented as the series over Hamiltonian eigenstates [40]:

$$
\begin{aligned}
C\left(n_{t}\right) & =\left\langle\psi^{\dagger}\left(\mathbf{0}, n_{t}\right) \Gamma_{1} \psi\left(\mathbf{0}, n_{t}\right) \psi^{\dagger}(\mathbf{0}, 0) \Gamma_{2} \psi(\mathbf{0}, 0)\right\rangle_{A} \\
& =\sum_{k}\langle 0|\hat{O}| k\rangle\left\langle k\left|\hat{O}^{\dagger}\right| 0\right\rangle e^{-n_{t} a E_{k}}
\end{aligned}
$$

where $E_{k}$ is the energy of the state with the quantum number $k, \hat{O}, \hat{O}^{\dagger}$ are the operators acting on the Hilbert space.

The correlator is the second rank tensor invariant w.r.t. Lorentz group transformation. It is, in fact, proportional to the vector meson spin covariant density matrix, which is especially convenient to consider in its rest frame. The symmetry is than reduced to rotational one and the irreducible representations include the scalar (corresponding to spinaveraged cross-section), axial vector (corresponding to 3 components of vector polarization) and symmetric traceless tensor (corresponding to 5 components of tensor polarization). The background magnetic field is directed along the $\mathrm{z}$ axis. It is convenient to express the spin density matrix in terms of transverse $\left(e_{x}=(0,1,0,0), e_{y}=(0,0,1,0)\right)$ and longitudinal $\left(e_{z}=(0,0,0,1)\right)$ polarization vectors. One can obtain the energy of the ground state for the spin projection $s_{z}=0$ on the field direction from equation (2.14), when $\Gamma_{1}, \Gamma_{2}=3$. The combinations of the correlators

$$
C\left(s_{z}= \pm 1\right)=C_{11}+C_{22} \pm i\left(C_{12}-C_{21}\right)
$$

give the energies of vector mesons with the spin projections equals to +1 and -1 correspondingly, indices ' 1 ' and '2' correspond to the $\gamma_{1}$ and $\gamma_{2}$ in (2.14) respectively.

At large $n_{t}$ the first term in formula (2.17), containing the energy of the ground state, gives the main contribution to the correlation function. Due to the boundary conditions it has the following form [40]:

$$
C_{f i t}\left(n_{t}\right)=2 A_{0} e^{-N_{T} a E_{0} / 2} \cosh \left(\left(n_{t}-\frac{N_{T}}{2}\right) a E_{0}\right)
$$




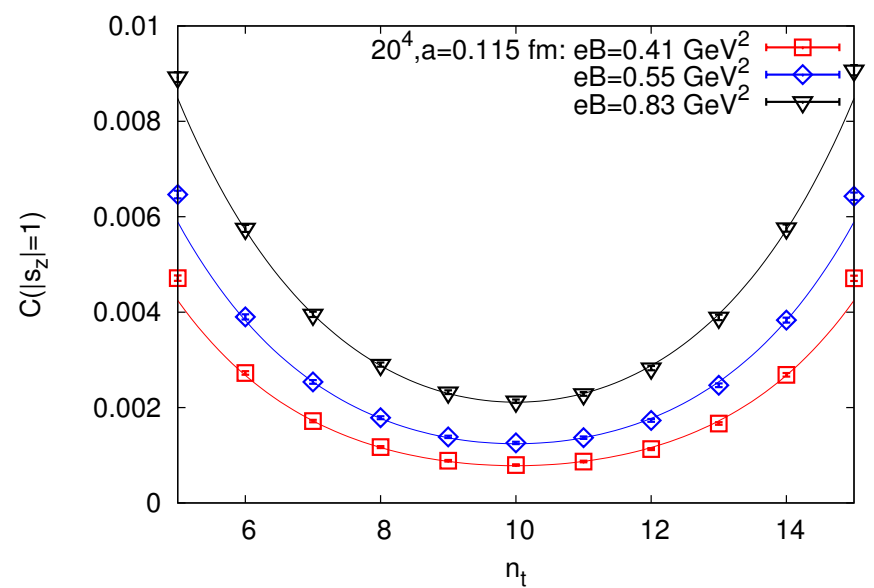

Figure 1. The correlation function of the $\rho^{+}\left(\rho^{-}\right)$meson with the $s_{z}=+1\left(s_{z}=-1\right)$ spin projection for $m_{\pi}(B=0)=535(4) \mathrm{MeV}$ and several values of the magnetic field. The curves are the fits of the lattice data made by the hyperbolic cosine function (2.19).

where $A_{0}$ is a constant, $E_{0}$ is the energy of the ground state. We find the ground state energy as the fit parameter, fitting lattice correlators by the function (2.19). In order to minimize the errors and exclude the contribution of excited states the data are fitted at $n_{0} \leq n_{t} \leq N_{T}-n_{0}$, where $n_{0}$ is the parameter which is determined from a plot of the effective mass. The errors are found from the fit of the correlation function by $\chi^{2}$ method.

The effective mass can be found from the formula

$$
m_{\mathrm{eff}}\left(n_{t}+\frac{1}{2}\right)=\ln \frac{C\left(n_{t}\right)}{C\left(n_{t}+1\right)},
$$

but it doesn't take into account the boundary conditions. To respects the periodicity one has to solve numerically the following equation

$$
\frac{C\left(n_{t}\right)}{C\left(n_{t}+1\right)}=\frac{\cosh \left(m_{\mathrm{eff}}\left(n_{t}-N_{T} / 2\right)\right)}{\cosh \left(m_{\mathrm{eff}}\left(n_{t}+1-N_{T} / 2\right)\right)}
$$

Figure 1 shows the correlation function of the $\rho^{+}\left(\rho^{-}\right)$meson with the $s_{z}=+1$ $\left(s_{z}=-1\right)$ spin projection on the magnetic field direction for the lattice volume $20^{4}$, the lattice spacing $0.115 \mathrm{fm}$, the pion mass $535(4) \mathrm{MeV}$ and several field values. Fits were done using the hyperbolic cosine function (2.19) at $n_{0}=6$. In figure 2 the plot of the effective mass are shown for the same lattice parameters and for the magnetic field values $0.14 \mathrm{GeV}^{2}$ and $0.55 \mathrm{GeV}^{2}$. The points represent the $m_{\text {eff }}$ values obtained from equation $(2.21)$, the lines correspond to the fits by a constant function performed at $n_{0}=6$. One can also use $n_{0}=5$, the energies obtained at $n_{0}=5$ and $n_{0}=6$ coincide within the errors.

In figure 3 we depict the effective mass plot of the $\rho^{+}\left(\rho^{-}\right)$meson for another spin projection $s_{z}=-1\left(s_{z}=+1\right)$ on the magnetic field direction. From a comparison of figure 2 and figure 3 we see that the errors of the mass determination are sufficiently larger for the highest energy sublevel than for the lowest sublevel. 


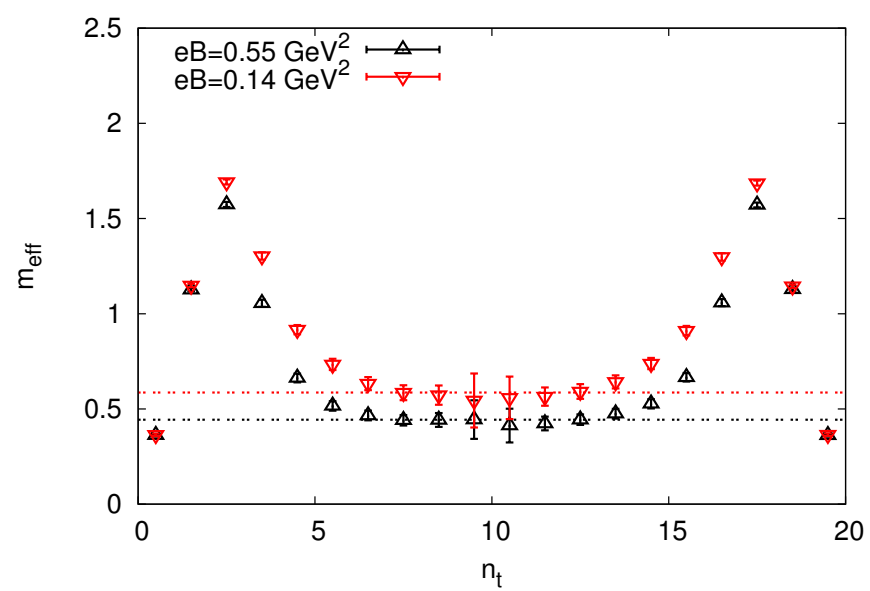

Figure 2. The effective mass of the $\rho^{+}\left(\rho^{-}\right)$meson with the $s_{z}=+1\left(s_{z}=-1\right)$ spin projection for $m_{\pi}(B=0)=535(4) \mathrm{MeV}$. The lines correspond to the fits of the $m_{\mathrm{eff}}$ by the constant function at $n_{0}=6$.

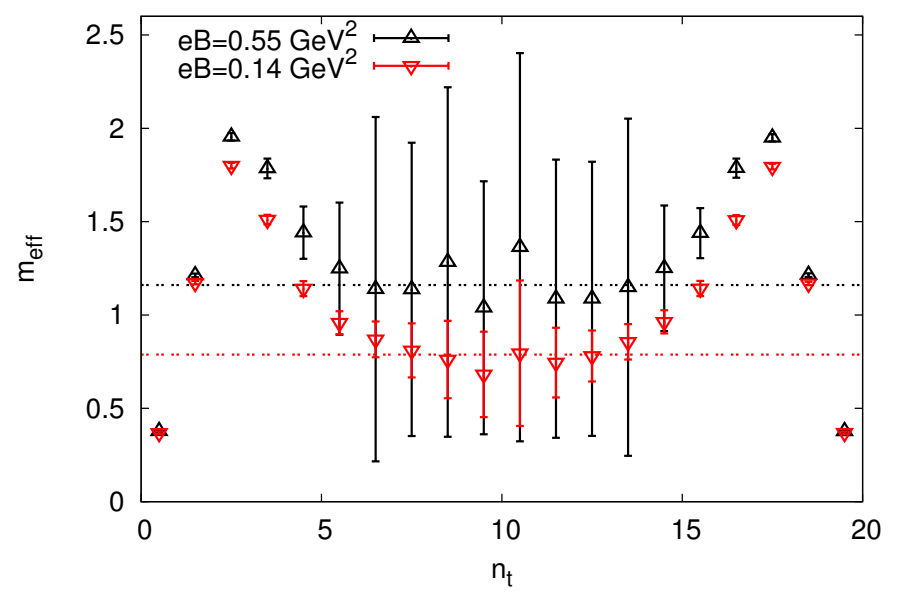

Figure 3. The effective mass of the $\rho^{+}\left(\rho^{-}\right)$meson with the $s_{z}=-1\left(s_{z}=+1\right)$ spin projection for $m_{\pi}(B=0)=535(4) \mathrm{MeV}$. The lines correspond to the fits of the $m_{\mathrm{eff}}$ by the constant function at $n_{0}=6$.

\section{Magnetic moments of $\rho^{ \pm}$and $K^{* \pm}$ mesons}

Let us estimate the Lande $g$-factor of the vector mesons from the lattice data, using its values we obtain the magnetic polarizabilities (section 5). The $g$-factor characterizes the gyromagnetic ratio of a particle or its magnetic moment in natural magnetons.

The vector $\rho^{ \pm}$and $K^{* \pm}$ mesons, consisting of the strongly interacting quarks and gluons, have a complex structure. The precise determination of the $g$-factor value is of much interest because it enables to find the contribution of non-perturbative effects to the magnetic moment of hadrons. However, the $\rho$ meson is an unstable and short-lived particle, so the experimental value of the $g$-factor is hard to measure.

The energy of the Landau levels is described by the following formula

$$
E^{2}=p_{z}^{2}+(2 n+1)|q B|-g s_{z} q B+m^{2},
$$




\begin{tabular}{|c|r|r|r|r|r|}
\hline$V$ & $m_{\pi}(\mathrm{MeV})$ & $a(\mathrm{fm})$ & $g$-factor & $\chi^{2} /$ d.o.f. & fit, $e B\left(\mathrm{GeV}^{2}\right)$ \\
\hline $18^{4}$ & $331 \pm 7$ & 0.115 & $2.01 \pm 0.18$ & 0.826 & {$[0,0.35]$} \\
\hline $18^{4}$ & $395 \pm 6$ & 0.115 & $2.17 \pm 0.18$ & 0.969 & {$[0,0.35]$} \\
\hline $18^{4}$ & $541 \pm 3$ & 0.115 & $2.12 \pm 0.07$ & 1.159 & {$[0,0.35]$} \\
\hline $18^{4}$ & $667 \pm 3$ & 0.115 & $2.07 \pm 0.19$ & 1.695 & {$[0,0.35]$} \\
\hline $18^{4}$ & $625 \pm 21$ & 0.086 & $2.11 \pm 0.01$ & 0.153 & {$[0,0.70]$} \\
\hline $18^{4}$ & $596 \pm 12$ & 0.095 & $2.30 \pm 0.12$ & 1.094 & {$[0,0.55]$} \\
\hline $18^{4}$ & $572 \pm 16$ & 0.105 & $2.05 \pm 0.03$ & 0.644 & {$[0,0.45]$} \\
\hline $20^{4}$ & $535 \pm 4$ & 0.115 & $2.22 \pm 0.08$ & 1.398 & {$[0,0.45]$} \\
\hline
\end{tabular}

Table 1. The g-factor of $\rho^{ \pm}$meson for the various lattice volumes, the pion masses and the lattice spacings, the nonrenormalized quark masses are also shown. The values of $\chi^{2} /$ d.o.f. and fitting intervals are also represented.

where $p_{z}$ is the momentum in the ' $\mathrm{z}$ ' spatial direction, $n$ is the principal quantum number, $q$ is the electric charge of the meson, $g$ is the g-factor, $s_{z}$ is the spin projection on the field direction and $m \equiv E(B=0)$ is the energy of the particle at zero magnetic field and zero momentum. Further we consider $n=0$ and $p_{z}=0$. This equation correspond to pint-like particle and should get the corrections from its structure for high mgnaetic fields.

At relatively low magnetic fields $\left(\leq 0.5 \mathrm{GeV}^{2}\right)$ the energy squared of the $\rho^{ \pm}$mesons iexhibits the linear responce of the field

$$
E^{2}=|q B|-g s_{z} q B+m^{2}
$$

One can neglect the nonlinear response on the magnetic field because the next term contribution to the energy at these $B$ values is less then $10 \%$ (see section 4 ). The energy of a meson can be described by a formula (3.2), because at the magnetic fields lower than QCD scale the internal structure of the meson has not to be revealed. It follows from equation (3.2), the energy splits into sublevels in the external magnetic field. The $E^{2}$ value increases at $q s_{z}=0,-1$ and decreases at $q s_{z}=+1$.

In figure 4 we show the energy squared of the $\rho^{+}\left(\rho^{-}\right)$meson depending on the field value with the spin projection $s_{z}=+1\left(s_{z}=-1\right)$ for the lattice volume $18^{4}$, the lattice spacings $0.086 \mathrm{fm}, 0.095 \mathrm{fm}, 0.115 \mathrm{fm}$ and various pion masses, the lowest pion mass is equal to $331(7) \mathrm{MeV}$ at $a=0.115 \mathrm{fm}$. For the lattice volume $20^{4}$ the calculations were carried out at the pion mass $m_{\pi}=535(4) \mathrm{MeV}$ and the lattice spacing $a=0.115 \mathrm{fm}$. In figure 4 the points represent the lattice data, the lines are the fits to the lattice results obtained with the use of formula (3.2).

We find the $g$-factor from the lowest energy sublevel, because the statistical errors are lower for the lowest energy sublevel than for the upper one, as we have seen in section 2. In table 1 we collect the $g$ values obtained from the fit (3.2), their errors, the lattice simulation parameters, the $\chi^{2} / n$.d.f. values and the field intervals used for the fitting. In figure 5 all $g$-factor values are represented depending on the pion mass squared. 


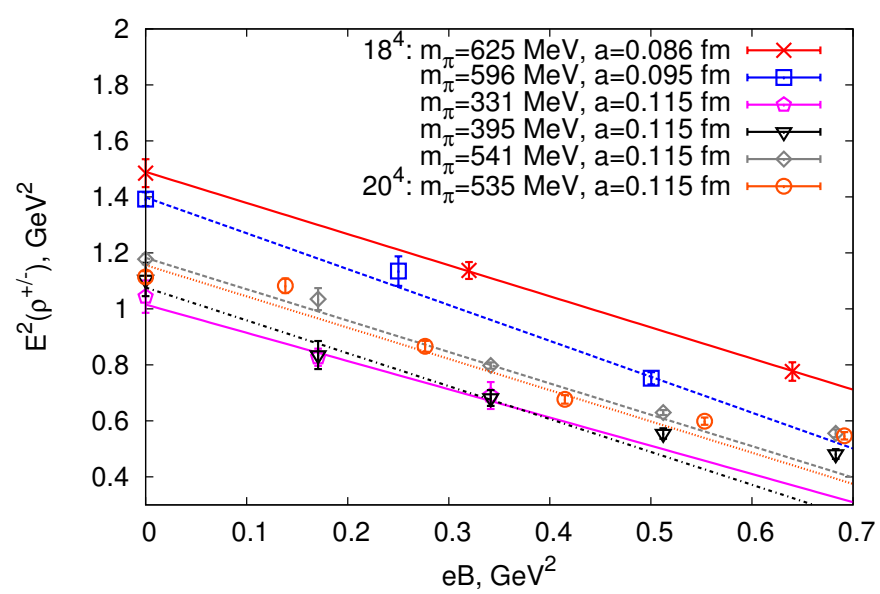

Figure 4. The energy squared of the $\rho^{+}\left(\rho^{-}\right)$meson with the spin projection $s_{z}=+1\left(s_{z}=-1\right)$ versus the magnetic field value for various lattice volumes, lattice spacings and different quark masses. The points correspond to the lattice data, the lines are the fits to these data obtained using formula (3.2).

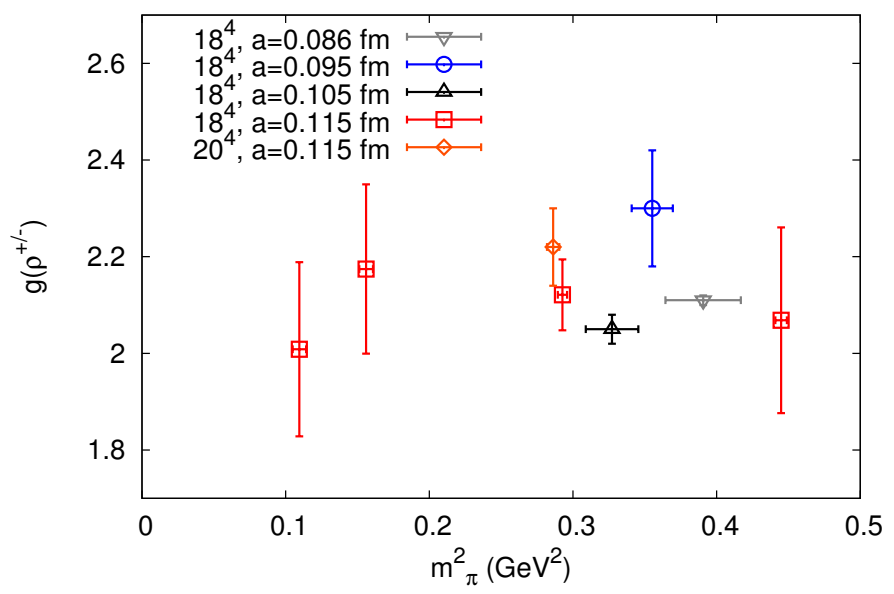

Figure 5. The g-factor of the charged $\rho$ meson versus the mass squared of the pion for various lattice data sets.

We consider several lattice spacings to check the cut-off effects, is there any $g$ value dependence on the lattice spacing. The cut-off effects, as well as energy dependence on the pion mass, are sizeable, as clearly visible in the figure 4 , where results from different simulations do not lie on top of each other. Nevertheless, we did not observe any dependence of the $g$-factor on the lattice spacing or the pion mass within the error range, the relative systematic error is $\sim 5 \%$ and a mean value is equal to $2.1 \pm 0.1_{\text {syst }}$. The finite volume effects are not so pronounced, for example lattices with the volumes $18^{4}$ and $20^{4}$, the lattice spacing $0.115 \mathrm{fm}$ give very close energy dependencies for the pion masses $541(3)$ and 535(4) respectively.

We have obtained the most accurate value $g=2.11 \pm 0.01$ for the finest lattice with the spacing $a=0.086 \mathrm{fm}$, the lattice volume $18^{4}$ and the pion mass $625(21) \mathrm{MeV}$. 


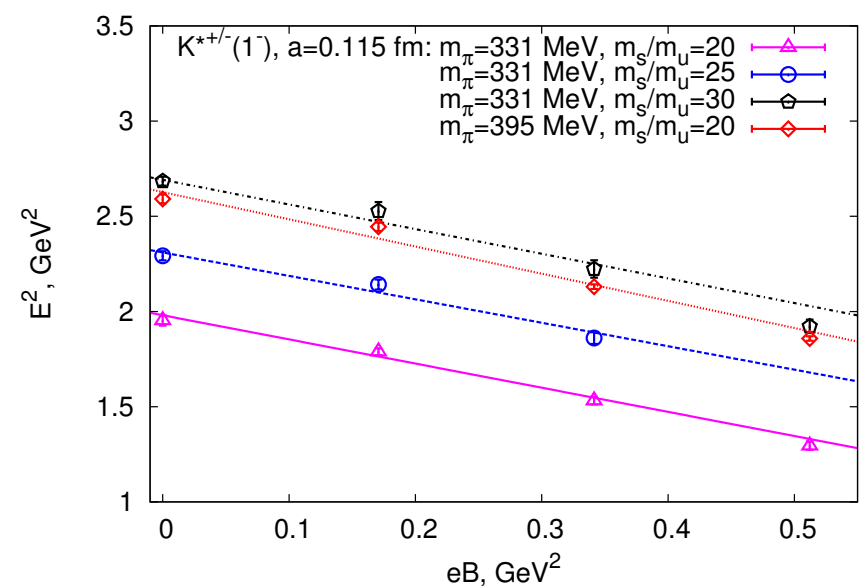

Figure 6. The energy squared of $K^{* \pm}$ meson with the spin projection $s_{z}= \pm 1$ versus the magnetic field value for the lattice volume $18^{4}$, the lattice spacing $0.115 \mathrm{fm}$ and various light and strange quark masses. Points correspond to the lattice data, lines are the fits to our data obtained using function (3.2).

\begin{tabular}{|c|r|r|r|r|}
\hline$m_{\pi}(\mathrm{MeV})$ & $m_{s} / m_{u}$ & $g$-factor & $\chi^{2} /$ d.o.f. & fit, $e B\left(\mathrm{GeV}^{2}\right)$ \\
\hline $331 \pm 7$ & 20 & $2.27 \pm 0.18$ & 1.845 & {$[0,0.35]$} \\
\hline $331 \pm 7$ & 25 & $2.23 \pm 0.23$ & 1.986 & {$[0,0.35]$} \\
\hline $331 \pm 7$ & 30 & $2.29 \pm 0.19$ & 1.366 & {$[0,0.35]$} \\
\hline
\end{tabular}

Table 2. The g-factor of the $K^{* \pm}$ meson, obtained on the lattice with the volume $18^{4}$, the lattice spacing $0.115 \mathrm{fm}$ and various bare masses of the light and strange quarks. The values of $\chi^{2} /$ d.o.f. and intervals choice for the fitting procedure are also shown.

Our value of the $\rho^{ \pm}$magnetic moment agrees with the other lattice predictions at nonzero pion masses $[27,30,41]$. The magnetic moment in pure gauge theory differs from the full $2+1$ QCD result, its value is lower for approximately $5-10 \%$ for the same pion masses [30]. This discrepancy may be explained by the contribution of the dynamical quarks, cut-off and finite volume effects.

D.G Gudino and G.T. Sanchez have obtained the g-factor from the analysis of BaBar cross section data for the reaction $e^{+} e^{-} \rightarrow \pi^{+} \pi^{-} 2 \pi^{0}$. They have found the value $g_{\exp }=$ $2.1 \pm 0.5$ [42]. The $g$-factor of the $\rho$ meson has been calculated in [29] using the chiral EFT at varying quark masses with a result similar to ours. The light cone QCD sum rules predict the value $g=2.4 \pm 0.4$ [43], and the covariant quark model gives $g=2.14$ [44].

Figure 6 shows the energy squared of $K^{* \pm}$ meson with the spin projection $s_{z}= \pm 1$ for the lightest pion mass $m_{\pi}=331(7) \mathrm{MeV}$, for the strange quark mass defined by the ratio $m_{s} / m_{u}=20,25,30$ and for pion mass $m_{\pi}=395(6) \mathrm{MeV}, m_{s}=20 m_{u}$. The fits to the lattice data, represented by the lines, were obtained by using formula (3.1). In table 2 the values of the g-factor for various light and strange quark masses are collected.

For the pion mass $m_{\pi}=331(7) \mathrm{MeV}$ and $m_{s}=30 m_{u}$, the closest to the physical case, the $g$-factor of the vector $K^{* \pm}$ meson is $2.29 \pm 0.19$. This value is in accordance with 
the prediction of the QCD sum rules $2.0 \pm 0.4$ [43] and the lattice results [27, 41]. Any dependence of the g-factor on the strange quark mass has not been observed within the error range.

The gyromagnetic ratio for free particles is close to "gravigyromagnetic" ratio, describing the motion of spin in rotating frames and near the rotating bodies (see [45] and ref. therein) which is universal $\left(g_{G}=2\right)$ due to equivalence principle. The value of $g \approx 2$ for $\rho$-meson shows that QCD effects do not strongly violate this similarity. One may also refer to AdS QCD where g is exactly equal to 2 [46].

\section{Energy of $\rho^{ \pm}$meson}

We have explored the ground state energy levels of the charged $\rho^{ \pm}$meson versus the value of the external magnetic field, which is directed along the ' $z$ ' axis.

Figure 7 shows the energy of the charged $\rho$ meson with the spin projections $s_{z}=-1,0$ and +1 on the direction of the magnetic field depending on the field value for the lattice volume $20^{4}$, the lattice spacing $0.115 \mathrm{fm}$ and the pion mass $m_{\pi}(B=0)=535(4) \mathrm{MeV}$. At the magnetic fields $e B \in[0,0.3] \mathrm{GeV}^{2}$ we fit the lattice data by formula (3.2). In figure 7 the corresponding fits are represented for the spin projections $s_{z}=-1,0$ and +1 by solid lines. In our calculations the reversal of the field direction is equivalent to the replacement of a quark by an antiquark, so the energy of the $\rho^{-}\left(s_{z}=-1\right)$ is identical to the energy of the $\rho^{+}\left(s_{z}=+1\right)$ meson.

At large magnetic fields the deviation from the Landau levels energy has been observed, because the energy sublevels of the $\rho$ meson are likely getting sensitive on the internal structure of the meson, which by definition is neglected when treating it as a free particle.

The parity has to be conserved, so for the spin projection $s_{z}=0$ on the field direction the $\rho$-meson energy squared contains only the terms of even powers of the magnetic field, while for the spin projections 1 and -1 the terms of both odd and even powers of the field are allowed.

In figure 7 we also show the fits of the lattice data (the dashed lines) by the following dependence

$$
E^{2}=|q B|-g s_{z} q B+m^{2}-4 \pi m \beta_{m}(q B)^{2}
$$

at $e B \in[0,1.2] \mathrm{GeV}^{2}$, where $\beta_{m}$ is the dipole magnetic polarizability.

One can include the next power of the field into consideration and fit the data for the $s_{z}= \pm 1$ by the formula

$$
E^{2}=|q B|-g s_{z} q B+m^{2}-4 \pi m \beta_{m}(q B)^{2}-4 \pi m \beta_{m}^{h 1}(q B)^{3},
$$

where $\beta_{m}^{h 1}$ is the magnetic hyperpolarizabilty of the first order. We have found that the fit coincides with the one obtained with the use of formula (4.1), the contribution of the cubic term in the field is negligible in comparison with the quadratic term at $e B \in[0,1.2]$. The term of the fourth power in the magnetic field gives even smaller contributions to the energy of the vector meson for the all spin projections at the field range considered, in this case the energy dependency is the following

$$
E^{2}=|q B|-g s_{z} q B+m^{2}-4 \pi m \beta_{m}(q B)^{2}-4 \pi m \beta_{m}^{h 1}(q B)^{3}-4 \pi m \beta_{m}^{h 2}(q B)^{4},
$$




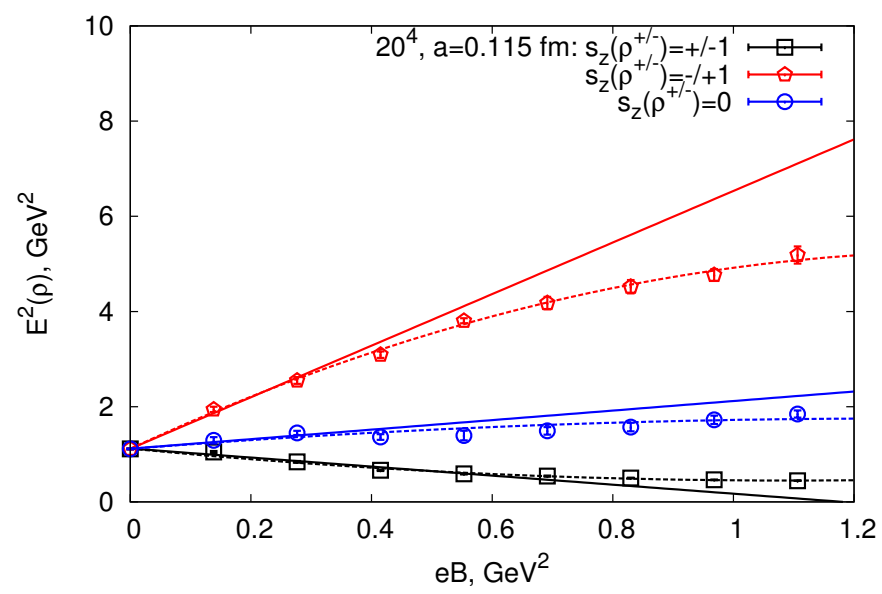

Figure 7. The energy squared of the charged $\rho$ meson for various spin projections $s_{z}=0, \pm 1$ depending on the magnetic field value for the lattice volume $V=20^{4}$, the lattice spacing $a=$ $0.115 \mathrm{fm}$ and the pion mass $m_{\pi^{0}}=535(4) \mathrm{MeV}$. The solid lines correspond to the fits of the lattice data obtained with the use of formula (3.2), the dashed lines are the fits of the lattice data by (4.1).

where $\beta_{m}^{h 2}$ is the magnetic hyperpolarizabilty of the second order. Thus, for the magnetic fields less than $1 \mathrm{GeV}^{2}$ the energy squared can be expanded in a series in the magnetic field. But at some value of the magnetic field the series of the perturbation theory in the magnetic field begins to diverge and the description in terms of the magnetic polarizabilities becomes poorly defined. One of the main objectives pursued in this work is to find a suitable range of field where the higher order effects can be neglected.

\section{Magnetic polarizabilities of $\rho$ meson}

The magnetic polarizabilities in QCD, whose example is represented by (4.2), quantify the response of an hadron to the strong magnetic field. In case of the lightest $\pi$ meson this quantity can be found from the ChPT. But if we deal with the magnetic polarizability of $\rho$ meson, any theoretical and experimental predictions are difficult to obtain. Thus the lattice QCD is a budding approach in this topic.

We have found the magnetic dipole polarizabilities fitting the lattice data by formula (4.1), where we fix the $g$-factor value, so that $m$ and $\beta_{m}$ are the fit parameters, $q s_{z}=+1$. The $g$-factor was found in section 3 and presented in table 1 . In figure 8 the solid line corresponds to the fit of the lattice data for the energy squared at the average value of the $g$-factor for the lattice volume $18^{4}$, the lattice spacing $a=0.115 \mathrm{fm}$ and the pion mass 395(6) MeV. The lattice data are shown by the empty points. The shaded area indicates the interval of the possible energy values, if we change the g-factor within the error range. The dashed lines correspond to the fits of the lattice data at $g=\bar{g} \pm \delta$, where $\delta$ is the error.

The values of the magnetic dipole polarizability are represented in table 3 , the errors include both the deviation of the $\beta_{m}$ from its average value due to the uncertainty of the $g$-factor and the error of the fitting procedure. For the lattice spacing $a=0.095 \mathrm{fm}$ we 


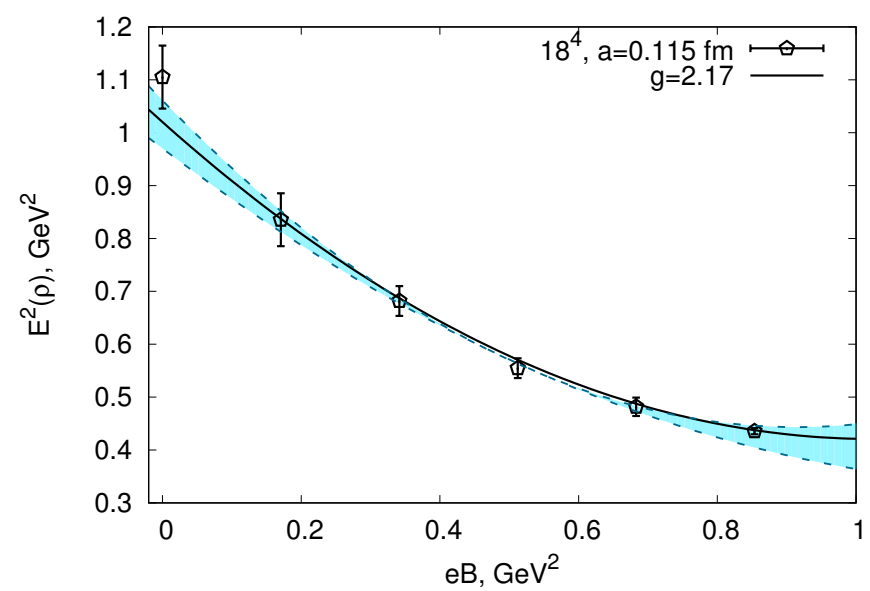

Figure 8. The energy squared of the ground state of the $\rho^{+}\left(\rho^{-}\right)$meson with the spin projection $s_{z}=+1\left(s_{z}=-1\right)$ versus the field value for the lattice spacing $a=0.115 \mathrm{fm}$, the lattice volume $18^{4}$ and the pion mass $395(6) \mathrm{MeV}$. The curve is the fit to the lattice data performed of formula (4.1). The shaded area represents the error of $E^{2}$ at the fixed $g$-factor value.

\begin{tabular}{|c|r|r|r|r|r|}
\hline$V$ & $m_{\pi}(\mathrm{MeV})$ & $a(\mathrm{fm})$ & $\beta_{m}\left(\mathrm{GeV}^{-3}\right)$ & $\chi^{2} /$ d.o.f. & fit, $e B\left(\mathrm{GeV}^{2}\right)$ \\
\hline $18^{4}$ & $596 \pm 12$ & 0.095 & $-0.025_{-0.014}^{+0.016}$ & 1.656 & {$[0,0.9]$} \\
\hline $18^{4}$ & $596 \pm 12$ & 0.095 & $-0.036_{-0.006}^{+0.007}$ & 1.864 & {$[0,1.3]$} \\
\hline $18^{4}$ & $541 \pm 3$ & 0.115 & $-0.037_{-0.005}^{+0.006}$ & 2.774 & {$[0,1.05]$} \\
\hline $20^{4}$ & $535 \pm 4$ & 0.115 & $-0.042_{-0.008}^{+0.008}$ & 2.274 & {$[0,1]$} \\
\hline $18^{4}$ & $395 \pm 6$ & 0.115 & $-0.045_{-0.012}^{+0.011}$ & 0.823 & {$[0,1]$} \\
\hline
\end{tabular}

Table 3. The magnetic dipole polarizabilty $\beta_{m}$ of the charged $\rho$ meson for the lattice spacings $0.095 \mathrm{fm}, 0.115 \mathrm{fm}$, for the lattice volume $18^{4}$, various pion masses and for the lattice spacing $0.115 \mathrm{fm}$, the lattice volume and the pion mass $535(4) \mathrm{MeV}$ with the corresponding errors and $\chi^{2} /$ d.o.f. The fit interval is shown in the last column. The results were obtained from the 2parametric fit according to formula (4.1).

show two intervals of the magnetic field which have been used for the determination of the polarizability, the results agree with each other within the errors.

Figure 9 shows that the values of the $g$-factor and the magnetic dipole polarizability $\beta_{m}$ slightly depend on the interval used for the fit procedure. With the increase of the interval the $\beta_{m}$ value starts to increase slowly from $e B \gtrsim 1 \mathrm{GeV}^{2}$. We explain this effect by the contribution of the magnetic hyperpolarizability $\beta_{m}^{1 h}(4.2)$, which gives an opposite contribution to the meson energy and not taken into account in equation (4.1).

We fit the energy squared using equation (4.2) to obtain the magnetic hyperpolarizability of the first order $\beta_{m}^{h 1}$. We can fix only the $g$-factor or the $g$-factor and the magnetic dipole polarizability $\beta_{m}$ at the same time. In the first case we deal with the 3-parametric fit, so that $m, \beta_{m}$ and $\beta_{m}^{h 1}$ are the fit parameters. In figure 10 the region of the acceptable $E^{2}$ values is restricted by the solid lines, the corresponding values of the $\beta_{m}$ and $\beta_{m}^{1 h}$ are shown in table 4 . 


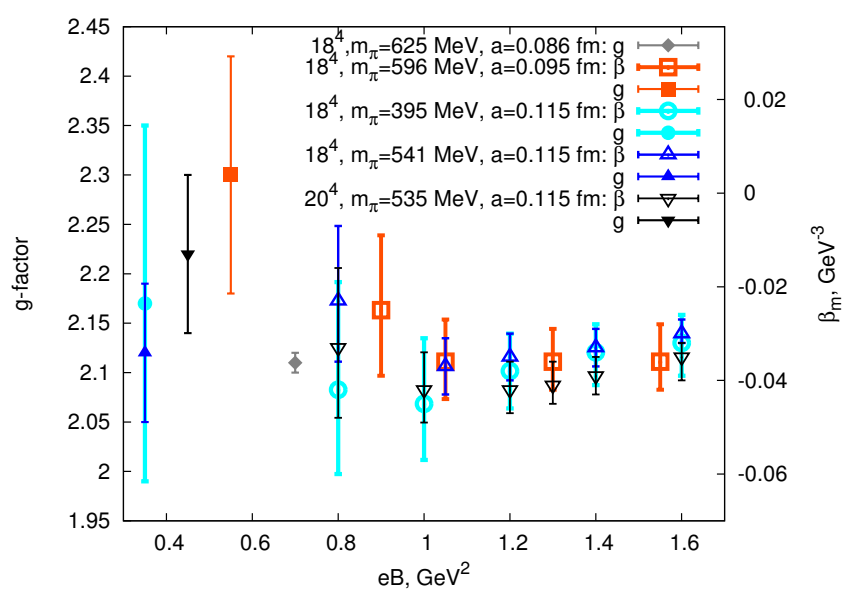

Figure 9. The $g$-factor and the dipole magnetic polarizability $\beta_{m}$ depending on the interval of fields used for their determination for various lattices and pion masses.

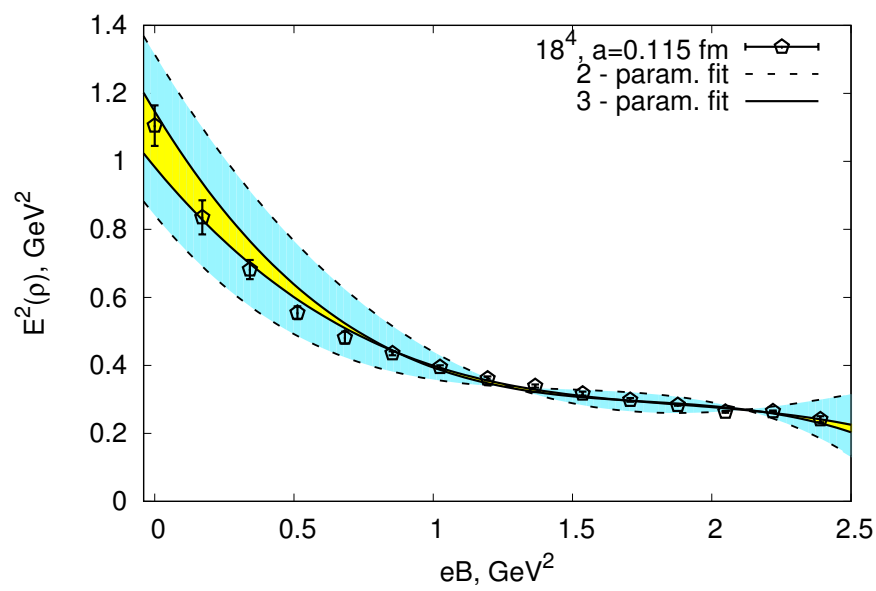

Figure 10. The energy squared of the ground state of the $\rho^{ \pm}$mesons with the spin projection $s_{z}= \pm 1$ versus the field value for the lattice spacing $a=0.115 \mathrm{fm}$, the lattice volume $18^{4}$ and the pion mass 395(6) MeV. The solid curves restrict the area obtained using the 3-parametric fit (4.2) at the fixed value of the $g$-factor. The dashed lines are the boundary of the acceptable $E^{2}$ values if the $g$-factor and $\beta_{m}$ have the definite values.

\begin{tabular}{|c|r|r|r|r|r|r|}
\hline$V$ & $m_{\pi}(\mathrm{MeV})$ & $a(\mathrm{fm})$ & $\beta_{m}\left(\mathrm{GeV}^{-3}\right)$ & $\beta_{m}^{1 h}\left(\mathrm{GeV}^{-5}\right)$ & $\chi^{2} /$ d.o.f. & $e B, \mathrm{GeV}^{2}$ \\
\hline $18^{4}$ & $596 \pm 12$ & 0.095 & $-0.050_{-0.008}^{+0.009}$ & $0.009_{-0.003}^{+0.002}$ & 1.965 & {$[0,2.5]$} \\
\hline $18^{4}$ & $541 \pm 3$ & 0.115 & $-0.045_{-0.005}^{+0.005}$ & $0.009_{-0.001}^{+0.001}$ & 2.787 & {$[0,2.5]$} \\
\hline $20^{4}$ & $535 \pm 4$ & 0.115 & $-0.058_{-0.008}^{+0.008}$ & $0.013_{-0.003}^{+0.002}$ & 2.697 & {$[0,2.0]$} \\
\hline $18^{4}$ & $395 \pm 6$ & 0.115 & $-0.047_{-0.009}^{+0.009}$ & $0.009_{-0.002}^{+0.002}$ & 2.255 & {$[0,2.5]$} \\
\hline
\end{tabular}

Table 4. The magnetic dipole polarizabilty $\beta_{m}$, the magnetic hyperpolarizability $\beta_{m}^{1 h}$ of the charged $\rho$ meson obtained from the 3 -parametric fit (4.2), where we use the data presented in table 1 . The results are obtained for various lattice volumes $V$, lattice spacings $a$ and pion masses $m_{\pi}, \chi^{2} /$ d.o.f. values correspond to the fit at the average $g$-factor value. 


\begin{tabular}{|c|r|r|r|r|}
\hline$V$ & $m_{\pi}(\mathrm{MeV})$ & $a(\mathrm{fm})$ & $\beta_{m}^{1 h}\left(\mathrm{GeV}^{-5}\right)$ & $e B, \mathrm{GeV}^{2}$ \\
\hline $18^{4}$ & $596 \pm 12$ & 0.095 & $0.004_{-0.002}^{+0.002}$ & {$[0,2.5]$} \\
\hline $18^{4}$ & $541 \pm 3$ & 0.115 & $0.006_{-0.002}^{+0.001}$ & {$[0,2.5]$} \\
\hline $20^{4}$ & $535 \pm 4$ & 0.115 & $0.006_{-0.002}^{+0.003}$ & {$[0,2.0]$} \\
\hline $18^{4}$ & $395 \pm 6$ & 0.115 & $0.008_{-0.004}^{+0.005}$ & {$[0,2.5]$} \\
\hline
\end{tabular}

Table 5. The magnetic hyperpolarizability $\beta_{m}^{1 h}$ of the charged $\rho$ meson obtained from the 2parametric fit (4.2), where we use the data from table 1 and table 4 . The results are obtained for various lattice volumes $V$, lattice spacings $a$ and pion masses $m_{\pi}$.

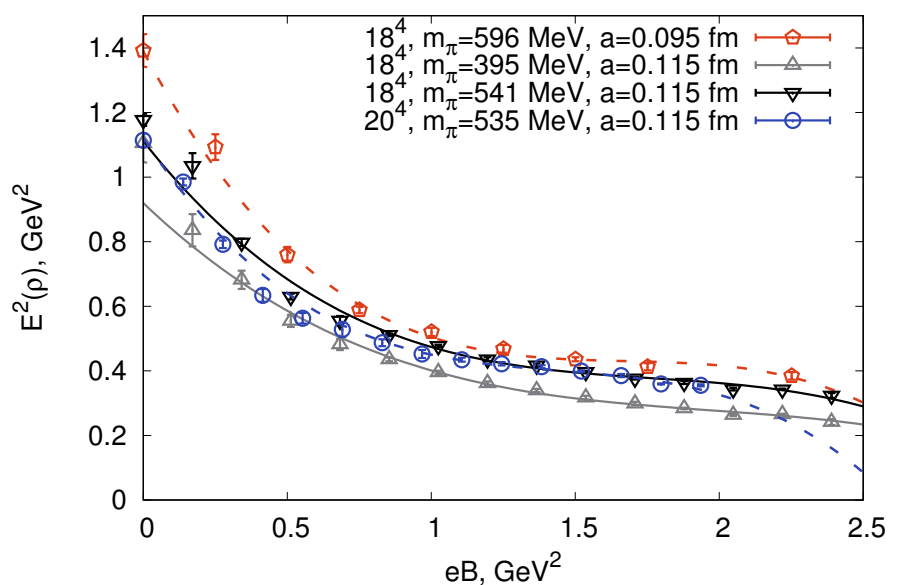

Figure 11. The energy squared of the ground state of the $\rho^{+}\left(\rho^{-}\right)$meson with the spin projection $s_{z}=+1\left(s_{z}=-1\right)$ versus the field value for various sets of lattice data. The curves are the fits to the lattice data obtained with the use of formula (4.2).

Zero hyperpolarizability of the second order $\beta_{m}^{h 2}$ was obtained within the errors fitting the data according to formula (4.3) at $e B \in[0,2.5] \mathrm{GeV}^{2}$, so we consider its contribution insignificant.

We also have found the magnetic hyperpolarizability $\beta_{m}^{h 1}$ from the 2-parametric fit (4.2) for a check, using the fixed $g$-factor from table 1 and the $\beta_{m}$ from table 3 . In figure 10 the region limited by the dashed lines corresponds to such fits if we change the $g$-factor and the magnetic dipole polarizability $\beta_{m}$ within their errors. The values of $\beta_{m}^{h 1}$ are presented in table 5 and agree with the results obtained with the use of the 3 -parametric fit.

At the value of the magnetic dipole polarizability $\sim 0.045 \mathrm{GeV}^{-3}$ the contribution of the quadratic term in field to the energy squared is approximately equal to $10 \%$ at $e B=0.35 \mathrm{GeV}^{2}$, that is at least not larger than the errors of the $g$-factor. At the magnetic field $\sim 1 \mathrm{GeV}^{2}$ the term with the hyperpolarizability gives the correction $\sim 20 \%$ to the term with the magnetic dipole polarizability, which is compatible with its errors. The term with hyperpolarizability partially compensates the quadratic term in field at the magnetic fields considered here.

In figure 11 the energy squared of the ground state of the vector $\rho^{ \pm}$meson with the spin projection $s_{z}= \pm 1$ is shown by points for the various lattice data sets. The solid and dashed 


\begin{tabular}{|c|r|r|r|r|r|r|}
\hline$V$ & $m_{\pi}(\mathrm{MeV})$ & $a(\mathrm{fm})$ & $g$-factor & $\beta_{m}\left(\mathrm{GeV}^{-3}\right)$ & $\beta_{m}^{1 h}\left(\mathrm{GeV}^{-5}\right)$ & $\chi^{2} /$ d.o.f. \\
\hline $18^{4}$ & $596 \pm 12$ & 0.095 & $2.49 \pm 0.18$ & $-0.056 \pm 0.008$ & $0.010 \pm 0.002$ & 3.738 \\
\hline $18^{4}$ & $541 \pm 3$ & 0.115 & $2.09 \pm 0.09$ & $-0.043 \pm 0.005$ & $0.008 \pm 0.001$ & 2.993 \\
\hline $20^{4}$ & $535 \pm 4$ & 0.115 & $2.35 \pm 0.11$ & $-0.067 \pm 0.008$ & $0.015 \pm 0.002$ & 2.657 \\
\hline $18^{4}$ & $395 \pm 6$ & 0.115 & $1.85 \pm 0.11$ & $-0.033 \pm 0.006$ & $0.006 \pm 0.001$ & 3.191 \\
\hline
\end{tabular}

Table 6. The magnetic dipole moment, the magnetic dipole polarizability and magnetic hyperpolarizability of the first order $\beta_{m}^{1 h}$ and second order $\beta_{m}^{2 h}$ of the charged $\rho$ meson for the lattice spacings $0.095 \mathrm{fm}, 0.115 \mathrm{fm}$, the lattice volume $18^{4}$, various pion masses and for the lattice spacing $0.115 \mathrm{fm}$, the lattice volume $20^{4}$ and the pion mass $m_{\pi}=535(4) \mathrm{MeV}$ with their errors and $\chi^{2} /$ d.o.f values. The results were obtained with the use of 4-parametric fit (4.2).

lines are the 4-parametric fits to the lattice data obtained with the use of formula (4.2), where $m, g, \beta_{m}$ and $\beta_{m}^{1 h}$ are the fit parameters presented in table 6 . These results agree with the previous results for the $g$-factor, magnetic polarizability and hyperpolarizability, but the $g$-factor values have bigger errors.

The nonlinear terms containing magnetic polarizabilities make the energy dependence rather flat at large magnetic fields, otherwise the lowest energy sublevel would cross zero at $e B \sim 1 \mathrm{GeV}^{2}$ as it follows from the Landau level picture (3.2). The higher order dependence of the energy on $B$ tends to be sign-changing from order to order and results in rather flat behaviour. This is quite important and constitutes one of the main results of the present paper.

The importance stems from the fact that the lowest order linear dependence leads to the node of the mass square of the $\rho^{-}\left(\rho^{+}\right)$meson for $s_{=}-1\left(s_{z}=1\right)$. This signals the appearance of tachyonic mode which may lead to superconducting phase transition and Abrikosov lattice appearance known for many years in electroweak theory [47]. The transition to superconducting phase was recently discovered and extensively studied in the actual case of $\rho$ mesons (See [48] and ref. therein).

We observe that the magnetic (hyper)polarizabilities provide the "self-healing" of such a behaviour. One may even speculate, that QCD prevents the phase transition and saves our Universe in the case of presence of even extremely large magnetic fields at early stage of its evolution. At the same time, one cannot exclude, that the phase transition may emerge in some other manner.

Figure 12 shows the energy squared of vector $\rho^{-}\left(\rho^{+}\right)$meson with the spin projection $s_{z}=+1\left(s_{z}=-1\right)$ as a function of the field value. The $E^{2}$ behaviour is discribed by formula (4.2) at $q s_{z}=-1$. According to the symmetries the absolute values of the hyperpolarizabilities are equal for the $s_{z}=-1$ and $s_{z}=+1$ cases, so the term of the fourth power in $B$ has to give a very small relative contribution to the energy at the considered range of fields.

The extrapolation errors also appear to be essentially larger for the highest energy sublevel with the spin orientation opposite to magnetic field direction resulting in the higher energy. As a result, the values of the magnetic moment and the (hyper)polarizability are 


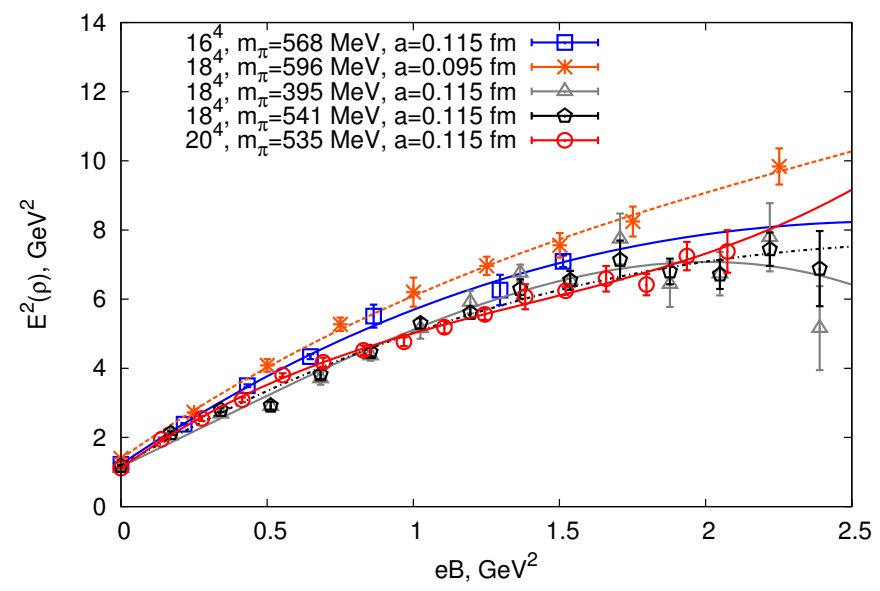

Figure 12. The energy squared of the ground state of $\rho^{-}\left(\rho^{+}\right)$meson with $s_{z}=+1\left(s_{z}=-1\right)$ depending on the magnetic field value for lattice volumes $16^{4}, 18^{4}, 20^{4}$, lattice spacings $0.095 \mathrm{fm}$, $0.115 \mathrm{fm}$ and various pion masses with the fits made using formula (4.2).

\begin{tabular}{|c|r|r|r|r|}
\hline$V$ & $m_{\pi}(\mathrm{MeV})$ & $a(\mathrm{fm})$ & $\beta_{m}\left(\mathrm{GeV}^{-3}\right)$ & $\chi^{2} /$ d.o.f. \\
\hline $18^{4}$ & $541 \pm 3$ & 0.115 & $0.026 \pm 0.004$ & 1.959 \\
\hline $20^{4}$ & $535 \pm 4$ & 0.115 & $0.026 \pm 0.005$ & 1.365 \\
\hline
\end{tabular}

Table 7. The magnetic dipole polarizability of the charged $\rho$ meson obtained for the lattice spacing $0.115 \mathrm{fm}$ and the lattice volumes $18^{4}, 20^{4}$ from the fit (5.1). The pion masses are represented in the second column, the values of $\chi^{2} /$ d.o. $f$ are shown in the last one.

compatible within errors for two energy sublevels, while the lower energy sublevel providing the smaller errors is used for obtaining final results for these physical quantities. One can see from figure 12 that there are no finite volume effects for this energy component, and energy data lie higher for $16^{4}$ lattice volume than for $18^{4}$ and $20^{4}$ due to a bit larger pion mass.

In figure 13 we show the energy squared of the charged $\rho$ meson with the $s_{z}=0$ versus the field value for various lattices. Due to the parity conservation the nonlinear terms in $B$ can give contributions to the $E^{2}$ value if they contain only even powers of the field. So, we fit our lattice data by the following formula

$$
E^{2}=|q B|+m^{2}-4 \pi m \beta_{m}(q B)^{2},
$$

at $e B \in[0,1.2] \mathrm{GeV}^{2}$, where $m, \beta_{m}$ are the fit parameters. Statistically significant values of polarizabilities were found for the lattices with the volumes $18^{4}$ and $20^{4}$, the lattice spacing $0.115 \mathrm{fm}$ and the quark mass $34.26 \mathrm{MeV}$, the results are shown in table 7 . For all the sublevels the energy of the $\rho$ meson is dependent on the lattice spacing and the pion mass.

Our results qualitatively disagree from the findings of [50], where at small value of B the energy of the lowest energy sublevel is observed to first decrease, then increase again. The possible explanation is that the overlap fermions do not break the full chiral symmetry in contrast to Wilson fermions. It enable to avoid the additional term for the renormalised 


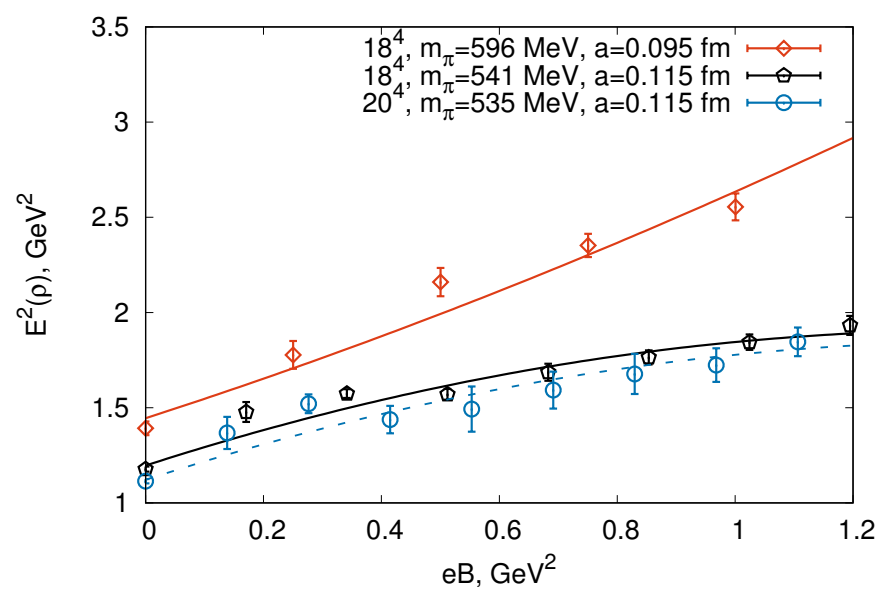

Figure 13. The energy squared of the ground state of the $\rho^{ \pm}$meson with the spin projection $s_{z}=0$ versus the field value for the lattice volumes $18^{4}$ and $20^{4}$, the lattice spacings $0.095 \mathrm{fm}$, $0.115 \mathrm{fm}$ and various pion masses $m_{\pi}$ with the corresponding fits (5.1).

quark mass which is not zero for Wilson fermions and depends on the lattice spacing and the magnetic field value $[5,51]$. Besides, the extraction procedure of the correlation functions for different spin projections is described in [50] briefly, so the detailed comparison is currently difficult to perform.

\section{Conclusion}

The energy levels of the ground state of vector $\rho^{ \pm}$and $K^{* \pm}$ mesons have been explored versus the magnetic field value in the $\mathrm{SU}(3)$ quenched lattice gauge theory. At low magnetic fields our data agrees with the picture of the Landau levels within the error range. At large magnetic fields $e B>[0.3 \div 0.5] \mathrm{GeV}^{2}$ the non-linear terms of the magnetic field give a contribution to the energy providing clear indications of the nonzero magnetic dipole polarizability and the hyperpolarizabilities. Our lattice data for the energy squared confirms the theoretical expectations.

We have calculated the magnetic dipole polarizability and hyperpolarizability of the charged $\rho$ mesons, while for the $s_{z}=0$ only the dipole polarizability has been found. For the low energy component we do not observe any significant dependence of the results on the lattice spacing and the lattice volume. But we expect these effects can appear for the highest energy level.

We have also found the g-factor of the vector $\rho^{ \pm}$and $K^{* \pm}$ mesons. The obtained values agree with the predictions of the QCD sum rules [43], the covariant quark model [44] and the lattice results at close pion masses $[27,41]$. The magnetic moment of the $\rho^{ \pm}$meson is in good agreement with the experimental result [42].

The magnetic dipole polarizability of the $\rho^{ \pm}$meson for the $s_{z}=-1$ and $s_{z}=+1$ energy components has to be the same, and we found it from the lowest energy sublevel with a good accuracy. Also we calculate the $\beta_{m}$ value for the $s_{z}=0$ case, it's opposite in sign and its absolute value is lower than for the $\left|s_{z}\right|=1$ for the same lattice data sets. 
The polarizability tensor of the $\rho^{ \pm}$meson at nonzero momentum is of a particular interest because it could be associated with the lepton asymmetry in a strong magnetic field [49].

It has been shown, that the meson energy doesn't turn to zero due to higher order dependence of the energy from B. The tachyonic mode does not seem to exist in QCD at zero temperature and chemical potential, and there is no respective condensation of $\rho$ mesons as it was predicted in some theoretical works, although one cannot exclude another mechanism of condensation.

\section{Acknowledgments}

We are thankful to Yu. A. Simonov and V.V. Skalozub for useful discussions. The authors are grateful to FAIR-ITEP supercomputer center where these numerical calculations were performed. This work is completely supported by a grant from the Russian Science Foundation (project number 16-12-10059).

\section{A Lattice parameters and values of the magnetic field}

Table 8. The lattice parameters such as the volume, the lattice spacing, the pion mass and the number of the lattice gauge configurations used for the calculations for the each value of the magnetic field. The value of the magnetic can be calculated according to equation (2.13), the negative value of $n_{B}$ corresponds to the negative projection of the magnetic field on the ' $z$ ' axis.

\begin{tabular}{|c|r|r|r|r|}
\hline$V$ & $a(\mathrm{fm})$ & $m_{\pi}(\mathrm{MeV})$ & $n_{B}$ & $N_{\text {conf }}$ \\
\hline $18^{4}$ & 0.086 & $625 \pm 21$ & 0 & 199 \\
\hline $18^{4}$ & 0.086 & $625 \pm 21$ & 1 & 199 \\
\hline $18^{4}$ & 0.086 & $625 \pm 21$ & 2 & 199 \\
\hline $18^{4}$ & 0.086 & $625 \pm 21$ & -2 & 198 \\
\hline $18^{4}$ & 0.086 & $625 \pm 21$ & -4 & 199 \\
\hline $18^{4}$ & 0.095 & $596 \pm 12$ & 0 & 198 \\
\hline $18^{4}$ & 0.095 & $596 \pm 12$ & 1 & 294 \\
\hline $18^{4}$ & 0.095 & $596 \pm 12$ & 2 & 295 \\
\hline $18^{4}$ & 0.095 & $596 \pm 12$ & 3 & 199 \\
\hline $18^{4}$ & 0.095 & $596 \pm 12$ & 4 & 198 \\
\hline $18^{4}$ & 0.095 & $596 \pm 12$ & 5 & 194 \\
\hline $18^{4}$ & 0.095 & $596 \pm 12$ & 6 & 196 \\
\hline $18^{4}$ & 0.095 & $596 \pm 12$ & 7 & 197 \\
\hline $18^{4}$ & 0.095 & $596 \pm 12$ & 8 & 198 \\
\hline $18^{4}$ & 0.095 & $596 \pm 12$ & 9 & 198 \\
\hline $18^{4}$ & 0.095 & $596 \pm 12$ & 10 & 198 \\
\hline $18^{4}$ & 0.095 & $596 \pm 12$ & 11 & 198 \\
\hline
\end{tabular}




\begin{tabular}{|c|r|r|r|r|}
\hline$V$ & $a(\mathrm{fm})$ & $m_{\pi}(\mathrm{MeV})$ & $n_{B}$ & $N_{\text {conf }}$ \\
\hline $18^{4}$ & 0.095 & $596 \pm 12$ & 12 & 197 \\
\hline $18^{4}$ & 0.095 & $596 \pm 12$ & 13 & 196 \\
\hline $18^{4}$ & 0.095 & $596 \pm 12$ & -1 & 295 \\
\hline $18^{4}$ & 0.095 & $596 \pm 12$ & -2 & 295 \\
\hline $18^{4}$ & 0.095 & $596 \pm 12$ & -4 & 199 \\
\hline $18^{4}$ & 0.095 & $596 \pm 12$ & -6 & 199 \\
\hline $18^{4}$ & 0.095 & $596 \pm 12$ & -8 & 198 \\
\hline $18^{4}$ & 0.095 & $596 \pm 12$ & -10 & 199 \\
\hline $18^{4}$ & 0.095 & $596 \pm 12$ & -12 & 197 \\
\hline $18^{4}$ & 0.095 & $596 \pm 12$ & -14 & 198 \\
\hline $18^{4}$ & 0.095 & $596 \pm 12$ & -16 & 198 \\
\hline $18^{4}$ & 0.095 & $596 \pm 12$ & -18 & 197 \\
\hline $18^{4}$ & 0.095 & $596 \pm 12$ & -20 & 198 \\
\hline $18^{4}$ & 0.095 & $596 \pm 12$ & -22 & 197 \\
\hline $18^{4}$ & 0.095 & $596 \pm 12$ & -24 & 199 \\
\hline $18^{4}$ & 0.095 & $596 \pm 12$ & -26 & 197 \\
\hline $18^{4}$ & 0.115 & $331 \pm 7$ & 0 & 336 \\
\hline $18^{4}$ & 0.115 & $331 \pm 7$ & 1 & 271 \\
\hline $18^{4}$ & 0.115 & $331 \pm 7$ & 2 & 337 \\
\hline $18^{4}$ & 0.115 & $331 \pm 7$ & 3 & 239 \\
\hline $18^{4}$ & 0.115 & $331 \pm 7$ & -2 & 277 \\
\hline $18^{4}$ & 0.115 & $331 \pm 7$ & -4 & 274 \\
\hline $18^{4}$ & 0.115 & $331 \pm 7$ & -6 & 238 \\
\hline $18^{4}$ & 0.115 & $395 \pm 6$ & 0 & 245 \\
\hline $18^{4}$ & 0.115 & $395 \pm 6$ & 1 & 235 \\
\hline $18^{4}$ & 0.115 & $395 \pm 6$ & 2 & 245 \\
\hline $18^{4}$ & 0.115 & $395 \pm 6$ & 3 & 332 \\
\hline $18^{4}$ & 0.115 & $395 \pm 6$ & 4 & 246 \\
\hline $18^{4}$ & 0.115 & $395 \pm 6$ & 5 & 250 \\
\hline $18^{4}$ & 0.115 & $395 \pm 6$ & 6 & 249 \\
\hline $18^{4}$ & 0.115 & $395 \pm 6$ & 7 & 250 \\
\hline $18^{4}$ & 0.115 & $395 \pm 6$ & 8 & 336 \\
\hline $18^{4}$ & 0.115 & $395 \pm 6$ & 9 & 336 \\
\hline $18^{4}$ & 0.115 & $395 \pm 6$ & 10 & 335 \\
\hline $18^{4}$ & 0.115 & $395 \pm 6$ & 11 & 248 \\
\hline $18^{4}$ & 0.115 & $395 \pm 6$ & 12 & 244 \\
\hline
\end{tabular}




\begin{tabular}{|c|r|r|r|r|}
\hline$V$ & $a(\mathrm{fm})$ & $m_{\pi}(\mathrm{MeV})$ & $n_{B}$ & $N_{\text {conf }}$ \\
\hline $18^{4}$ & 0.115 & $395 \pm 6$ & 13 & 249 \\
\hline $18^{4}$ & 0.115 & $395 \pm 6$ & 14 & 250 \\
\hline $18^{4}$ & 0.115 & $395 \pm 6$ & -2 & 249 \\
\hline $18^{4}$ & 0.115 & $395 \pm 6$ & -4 & 250 \\
\hline $18^{4}$ & 0.115 & $395 \pm 6$ & -6 & 311 \\
\hline $18^{4}$ & 0.115 & $395 \pm 6$ & -8 & 198 \\
\hline $18^{4}$ & 0.115 & $395 \pm 6$ & -10 & 241 \\
\hline $18^{4}$ & 0.115 & $395 \pm 6$ & -12 & 232 \\
\hline $18^{4}$ & 0.115 & $395 \pm 6$ & -14 & 238 \\
\hline $18^{4}$ & 0.115 & $395 \pm 6$ & -16 & 320 \\
\hline $18^{4}$ & 0.115 & $395 \pm 6$ & -18 & 333 \\
\hline $18^{4}$ & 0.115 & $395 \pm 6$ & -20 & 336 \\
\hline $18^{4}$ & 0.115 & $395 \pm 6$ & -22 & 248 \\
\hline $18^{4}$ & 0.115 & $395 \pm 6$ & -24 & 247 \\
\hline $18^{4}$ & 0.115 & $395 \pm 6$ & -26 & 248 \\
\hline $18^{4}$ & 0.115 & $395 \pm 6$ & -28 & 245 \\
\hline $18^{4}$ & 0.115 & $541 \pm 3$ & 0 & 294 \\
\hline $18^{4}$ & 0.115 & $541 \pm 3$ & 1 & 335 \\
\hline $18^{4}$ & 0.115 & $541 \pm 3$ & 2 & 335 \\
\hline $18^{4}$ & 0.115 & $541 \pm 3$ & 3 & 249 \\
\hline $18^{4}$ & 0.115 & $541 \pm 3$ & 4 & 304 \\
\hline $18^{4}$ & 0.115 & $541 \pm 3$ & 5 & 298 \\
\hline $18^{4}$ & 0.115 & $541 \pm 3$ & 6 & 248 \\
\hline $18^{4}$ & 0.115 & $541 \pm 3$ & 7 & 298 \\
\hline $18^{4}$ & 0.115 & $541 \pm 3$ & 8 & 299 \\
\hline $18^{4}$ & 0.115 & $541 \pm 3$ & 9 & 300 \\
\hline $18^{4}$ & 0.115 & $541 \pm 3$ & 10 & 299 \\
\hline $18^{4}$ & 0.115 & $541 \pm 3$ & 11 & 248 \\
\hline $18^{4}$ & 0.115 & $541 \pm 3$ & 12 & 250 \\
\hline $18^{4}$ & 0.115 & $541 \pm 3$ & 13 & 249 \\
\hline $18^{4}$ & 0.115 & $541 \pm 3$ & 14 & 250 \\
\hline $18^{4}$ & 0.115 & $541 \pm 3$ & -1 & 213 \\
\hline $18^{4}$ & 0.115 & $541 \pm 3$ & -2 & 250 \\
\hline $18^{4}$ & 0.115 & $541 \pm 3$ & -3 & 250 \\
\hline $18^{4}$ & 0.115 & $541 \pm 3$ & -4 & 335 \\
\hline $18^{4}$ & 0.115 & $541 \pm 3$ & -5 & 250 \\
\hline
\end{tabular}




\begin{tabular}{|c|r|r|r|r|}
\hline$V$ & $a(\mathrm{fm})$ & $m_{\pi}(\mathrm{MeV})$ & $n_{B}$ & $N_{\text {conf }}$ \\
\hline $18^{4}$ & 0.115 & $541 \pm 3$ & -6 & 249 \\
\hline $18^{4}$ & 0.115 & $541 \pm 3$ & -7 & 250 \\
\hline $18^{4}$ & 0.115 & $541 \pm 3$ & -8 & 309 \\
\hline $18^{4}$ & 0.115 & $541 \pm 3$ & -9 & 234 \\
\hline $18^{4}$ & 0.115 & $541 \pm 3$ & -10 & 297 \\
\hline $18^{4}$ & 0.115 & $541 \pm 3$ & -11 & 250 \\
\hline $18^{4}$ & 0.115 & $541 \pm 3$ & -12 & 249 \\
\hline $18^{4}$ & 0.115 & $541 \pm 3$ & -13 & 250 \\
\hline $18^{4}$ & 0.115 & $541 \pm 3$ & -14 & 298 \\
\hline $18^{4}$ & 0.115 & $541 \pm 3$ & -16 & 300 \\
\hline $18^{4}$ & 0.115 & $541 \pm 3$ & -18 & 300 \\
\hline $18^{4}$ & 0.115 & $541 \pm 3$ & -20 & 298 \\
\hline $18^{4}$ & 0.115 & $541 \pm 3$ & -22 & 249 \\
\hline $18^{4}$ & 0.115 & $541 \pm 3$ & -24 & 250 \\
\hline $18^{4}$ & 0.115 & $541 \pm 3$ & -26 & 250 \\
\hline $18^{4}$ & 0.115 & $541 \pm 3$ & -28 & 247 \\
\hline $18^{4}$ & 0.115 & $667 \pm 3$ & 0 & 290 \\
\hline $18^{4}$ & 0.115 & $667 \pm 3$ & 1 & 195 \\
\hline $18^{4}$ & 0.115 & $667 \pm 3$ & 2 & 196 \\
\hline $18^{4}$ & 0.115 & $667 \pm 3$ & 3 & 266 \\
\hline $18^{4}$ & 0.115 & $667 \pm 3$ & 4 & 199 \\
\hline $18^{4}$ & 0.115 & $667 \pm 3$ & -1 & 198 \\
\hline $18^{4}$ & 0.115 & $667 \pm 3$ & -2 & 199 \\
\hline $18^{4}$ & 0.115 & $667 \pm 3$ & -3 & 300 \\
\hline $18^{4}$ & 0.115 & $667 \pm 3$ & -4 & 197 \\
\hline $20^{4}$ & 0.115 & $535 \pm 4$ & 0 & 290 \\
\hline $20^{4}$ & 0.115 & $535 \pm 4$ & 1 & 299 \\
\hline $20^{4}$ & 0.115 & $535 \pm 4$ & 2 & 295 \\
\hline $20^{4}$ & 0.115 & $535 \pm 4$ & 3 & 291 \\
\hline $20^{4}$ & 0.115 & $535 \pm 4$ & 4 & 296 \\
\hline $20^{4}$ & 0.115 & $535 \pm 4$ & 5 & 198 \\
\hline $20^{4}$ & 0.115 & $535 \pm 4$ & 6 & 296 \\
\hline $20^{4}$ & 0.115 & $535 \pm 4$ & 7 & 289 \\
\hline $20^{4}$ & 0.115 & $535 \pm 4$ & 8 & 298 \\
\hline $20^{4}$ & 0.115 & $535 \pm 4$ & 9 & 287 \\
\hline $20^{4}$ & 0.115 & $535 \pm 4$ & 10 & 294 \\
\hline
\end{tabular}




\begin{tabular}{|c|r|r|r|r|}
\hline$V$ & $a(\mathrm{fm})$ & $m_{\pi}(\mathrm{MeV})$ & $n_{B}$ & $N_{\text {conf }}$ \\
\hline $20^{4}$ & 0.115 & $535 \pm 4$ & 11 & 100 \\
\hline $20^{4}$ & 0.115 & $535 \pm 4$ & 12 & 100 \\
\hline $20^{4}$ & 0.115 & $535 \pm 4$ & 13 & 100 \\
\hline $20^{4}$ & 0.115 & $535 \pm 4$ & 14 & 100 \\
\hline $20^{4}$ & 0.115 & $535 \pm 4$ & -2 & 278 \\
\hline $20^{4}$ & 0.115 & $535 \pm 4$ & -4 & 295 \\
\hline $20^{4}$ & 0.115 & $535 \pm 4$ & -6 & 295 \\
\hline $20^{4}$ & 0.115 & $535 \pm 4$ & -8 & 256 \\
\hline $20^{4}$ & 0.115 & $535 \pm 4$ & -10 & 252 \\
\hline $20^{4}$ & 0.115 & $535 \pm 4$ & -12 & 300 \\
\hline $20^{4}$ & 0.115 & $535 \pm 4$ & -14 & 298 \\
\hline $20^{4}$ & 0.115 & $535 \pm 4$ & -16 & 300 \\
\hline $20^{4}$ & 0.115 & $535 \pm 4$ & -18 & 300 \\
\hline $20^{4}$ & 0.115 & $535 \pm 4$ & -20 & 300 \\
\hline $20^{4}$ & 0.115 & $535 \pm 4$ & -22 & 100 \\
\hline $20^{4}$ & 0.115 & $535 \pm 4$ & -24 & 99 \\
\hline $20^{4}$ & 0.115 & $535 \pm 4$ & -26 & 100 \\
\hline $20^{4}$ & 0.115 & $535 \pm 4$ & -28 & 96 \\
\hline
\end{tabular}

Open Access. This article is distributed under the terms of the Creative Commons Attribution License (CC-BY 4.0), which permits any use, distribution and reproduction in any medium, provided the original author(s) and source are credited.

\section{References}

[1] V. Skokov, A. Yu. Illarionov and V. Toneev, Estimate of the magnetic field strength in heavy-ion collisions, Int. J. Mod. Phys. A 24 (2009) 5925 [arXiv:0907.1396] [INSPIRE].

[2] G. Martinelli, G. Parisi, R. Petronzio and F. Rapuano, The Proton and Neutron Magnetic Moments in Lattice QCD, Phys. Lett. B 116 (1982) 434 [INSPIRE].

[3] M. D'Elia, S. Mukherjee and F. Sanfilippo, QCD Phase Transition in a Strong Magnetic Background, Phys. Rev. D 82 (2010) 051501 [arXiv:1005.5365] [INSPIRE].

[4] M. D'Elia, Lattice QCD with purely imaginary sources at zero and non-zero temperature, PoS (LATTICE2014) 020 [arXiv: 1502 .06047] [INSPIRE].

[5] B.B. Brandt, G. Bali, G. Endrödi and B. Glässle, QCD spectroscopy and quark mass renormalisation in external magnetic fields with Wilson fermions, PoS (LATTICE 2015) 265 [arXiv: 1510.03899] [INSPIRE].

[6] E.V. Luschevskaya, O.E. Solovjeva, O.A. Kochetkov and O.V. Teryaev, Magnetic polarizabilities of light mesons in $\mathrm{SU}(3)$ lattice gauge theory, Nucl. Phys. B 898 (2015) 627 [arXiv: 1411.4284] [INSPIRE]. 
[7] E.V. Luschevskaya, O.A. Kochetkov, O.V. Teryaev and O.E. Solovjeva, $\pi^{ \pm}$and $\rho^{0, \pm}$ mesons in a strong magnetic field on the lattice, JETP Lett. 101 (2015) 674 [INSPIRE].

[8] NPLQCD collaboration, S.R. Beane et al., Ab initio Calculation of the $n p \rightarrow d \gamma$ Radiative Capture Process, Phys. Rev. Lett. 115 (2015) 132001 [arXiv:1505.02422] [InSPIRE].

[9] M.A. Andreichikov, B.O. Kerbikov, V.D. Orlovsky and Yu. A. Simonov, Meson Spectrum in Strong Magnetic Fields, Phys. Rev. D 87 (2013) 094029 [arXiv:1304.2533] [InSPIRE].

[10] H. Liu, L. Yu and M. Huang, Charged and neutral vector $\rho$ mesons in a magnetic field, Phys. Rev. D 91 (2015) 014017 [arXiv: 1408.1318] [INSPIRE].

[11] H. Taya, Hadron Masses in Strong Magnetic Fields, Phys. Rev. D 92 (2015) 014038 [arXiv: 1412.6877] [INSPIRE].

[12] M. Kawaguchi and S. Matsuzaki, Vector meson masses from a hidden local symmetry in a constant magnetic field, Phys. Rev. D 93 (2016) 125027 [arXiv:1511.06990] [InSPIRE].

[13] K. Hattori, T. Kojo and N. Su, Mesons in strong magnetic fields: (I) General analyses, Nucl. Phys. A 951 (2016) 1 [arXiv:1512.07361] [INSPIRE].

[14] S. Cho, K. Hattori, S.H. Lee, K. Morita and S. Ozaki, Charmonium Spectroscopy in Strong Magnetic Fields by QCD Sum Rules: S-Wave Ground States, Phys. Rev. D 91 (2015) 045025 [arXiv: 1411.7675] [INSPIRE].

[15] P. Gubler, K. Hattori, S.H. Lee, M. Oka, S. Ozaki and K. Suzuki, D mesons in a magnetic field, Phys. Rev. D 93 (2016) 054026 [arXiv: 1512.08864] [INSPIRE].

[16] A. Klein, Low-Energy Theorems for Renormalizable Field Theories, Phys. Rev. 99 (1955) 998 [INSPIRE].

[17] A.M. Baldin, Polarizability of nucleons, Nucl. Phys. 18 (1960) 310.

[18] Yu. M. Antipov et al., Measurement of $\pi^{-}-x M e s o n$ Polarizability in Pion Compton Effect, Phys. Lett. B 121 (1983) 445 [INSPIRE].

[19] L.V. Fil'kov and V.L. Kashevarov, Determination of pit- meson polarizabilities from the $\gamma \gamma \rightarrow \pi^{+} \pi^{-}$process, Phys. Rev. C 73 (2006) 035210 [nucl-th/0512047] [INSPIRE].

[20] COMPASS collaboration, C. Adolph et al., Measurement of the charged-pion polarizability, Phys. Rev. Lett. 114 (2015) 062002 [arXiv: 1405.6377] [INSPIRE].

[21] J. Gasser, M.A. Ivanov and M.E. Sainio, Low-energy photon-photon collisions to two loops revisited, Nucl. Phys. B 728 (2005) 31 [hep-ph/0506265] [INSPIRE].

[22] A. Aleksejevs and S. Barkanova, Hadron Structure in Chiral Perturbation Theory, Nucl. Phys. Proc. Suppl. 245 (2013) 17 [arXiv: 1309.3313] [INSPIRE].

[23] E.V. Luschevskaya, O.E. Solovjeva and O.V. Teryaev, Magnetic polarizability of pion, Phys. Lett. B 761 (2016) 393 [arXiv:1511.09316] [INSPIRE].

[24] W. Andersen and W. Wilcox, Lattice charge overlap. 1. Elastic limit of pi and rho mesons, Annals Phys. 255 (1997) 34 [hep-lat/9502015] [INSPIRE].

[25] A. Samsonov, Magnetic moment of the rho meson in QCD sum rules: $\alpha_{s}$ corrections, JHEP 12 (2003) 061 [hep-ph/0308065] [INSPIRE].

[26] V.V. Braguta and A.I. Onishchenko, rho meson form-factors and QCD sum rules, Phys. Rev. D 70 (2004) 033001 [hep-ph/0403258] [INSPIRE]. 
[27] J.N. Hedditch, W. Kamleh, B.G. Lasscock, D.B. Leinweber, A.G. Williams and J.M. Zanotti, Pseudoscalar and vector meson form-factors from lattice QCD, Phys. Rev. D 75 (2007) 094504 [hep-lat/0703014] [INSPIRE].

[28] V.D. Orlovsky and Yu. A. Simonov, Nambu-Goldstone mesons in strong magnetic field, JHEP 09 (2013) 136 [arXiv: 1306.2232] [INSPIRE].

[29] D. Djukanovic, E. Epelbaum, J. Gegelia and U.G. Meissner, The magnetic moment of the

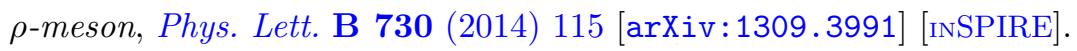

[30] B. Owen, W. Kamleh, D. Leinweber, B. Menadue and S. Mahbub, Light Meson Form Factors at near Physical Masses, Phys. Rev. D 91 (2015) 074503 [arXiv:1501.02561] [INSPIRE].

[31] H. Neuberger, Exactly massless quarks on the lattice, Phys. Lett. B 417 (1998) 141 [hep-lat/9707022] [INSPIRE].

[32] L. Giusti, C. Hölbling, M. Lüscher and H. Wittig, Numerical techniques for lattice QCD in the $\epsilon$-regime, Comput. Phys. Commun. 153 (2003) 31 [hep-lat/0212012] [INSPIRE].

[33] H. Neff, N. Eicker, T. Lippert, J.W. Negele and K. Schilling, On the low fermionic eigenmode dominance in QCD on the lattice, Phys. Rev. D 64 (2001) 114509 [hep-lat/0106016] [INSPIRE].

[34] M. Lüscher and P. Weisz, On-Shell Improved Lattice Gauge Theories, Commun. Math. Phys. 97 (1985) 59 [Erratum ibid. 98 (1985) 433] [INSPIRE].

[35] V.G. Bornyakov, E.M. Ilgenfritz and M. Müller-Preussker, Universality check of Abelian monopoles, Phys. Rev. D 72 (2005) 054511 [hep-lat/0507021] [InSPIRE].

[36] M.H. Al-Hashimi and U.J. Wiese, Discrete Accidental Symmetry for a Particle in a Constant Magnetic Field on a Torus, Annals Phys. 324 (2009) 343 [arXiv:0807.0630] [INSPIRE].

[37] G. 't Hooft, A Property of Electric and Magnetic Flux in Nonabelian Gauge Theories, Nucl. Phys. B 153 (1979) 141 [inSPIRE].

[38] H. Zainuddin, Group theoretic quantization of a particle on a torus in a constant magnetic field, Phys. Rev. D 40 (1989) 636 [inSPIRE].

[39] G.-H. Chen et al., Degeneracy of Landau levels and quantum qroup $s l_{q}(2)$, Phys. Rev. B 53 (1996) 9540.

[40] C. Gattringer and C.B. Lang, Quantum Chromodynamics on the Lattice, Lect. Notes Phys. 788, Springer-Verlag Berlin Heidelberg (2010).

[41] F.X. Lee, S. Moerschbacher and W. Wilcox, Magnetic moments of vector, axial and tensor mesons in lattice QCD, Phys. Rev. D 78 (2008) 094502 [arXiv:0807.4150] [INSPIRE].

[42] D. García Gudiño and G. Toledo Sánchez, Determination of the magnetic dipole moment of the rho meson using 4 pion electroproduction data, Int. J. Mod. Phys. Conf. Ser. 35 (2014) 1460463 [arXiv: 1305.6345] [INSPIRE].

[43] T.M. Aliev, A. Özpineci and M. Savci, Magnetic and quadrupole moments of light spin-1 mesons in light cone QCD sum rules, Phys. Lett. B 678 (2009) 470 [arXiv:0902.4627] [INSPIRE].

[44] J.P. B.C. de Melo and T. Frederico, Covariant and light front approaches to the rho meson electromagnetic form-factors, Phys. Rev. C 55 (1997) 2043 [nucl-th/9706032] [INSPIRE].

[45] O.V. Teryaev, Gravitational form factors and nucleon spin structure, Front. Phys. (Beijing) 11 (2016) 111207 [INSPIRE]. 
[46] H.R. Grigoryan and A.V. Radyushkin, Form Factors and Wave Functions of Vector Mesons in Holographic QCD, Phys. Lett. B 650 (2007) 421 [hep-ph/0703069] [INSPIRE].

[47] V.V. Skalozub, Abrikosov's lattice in the theory of electroweak interactions (in Russian), Yad. Fiz. 43 (1986) 1045 [INSPIRE].

[48] M.N. Chernodub, Superconductivity of QCD vacuum in strong magnetic field, Phys. Rev. D 82 (2010) 085011 [arXiv: 1008.1055] [INSPIRE].

[49] P.V. Buividovich, M.I. Polikarpov and O.V. Teryaev, Lattice studies of magnetic phenomena in heavy-ion collisions, Lect. Notes Phys. 871 (2013) 377 [arXiv:1211.3014] [INSPIRE].

[50] Y. Hidaka and A. Yamamoto, Charged vector mesons in a strong magnetic field, Phys. Rev. D 87 (2013) 094502 [arXiv: 1209.0007] [INSPIRE].

[51] G.S. Bali, B.B. Brandt, G. Endrödi and B. Glässle, Meson masses in electromagnetic fields with Wilson fermions, arXiv:1707.05600 [INSPIRE]. 